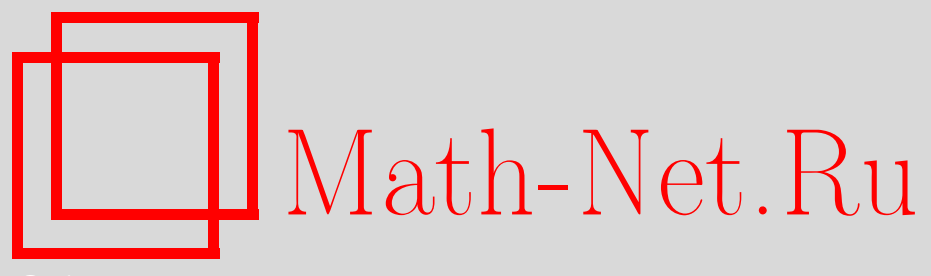

В. А. Кыров, Критерий невырожденности группы преобразований, Матем. заметки, 2009, том 85, выпуск 1, 144-146

DOI: https://doi.org/10.4213/mzm4993

Использование Общероссийского математического портала Math-Net.Ru подразумевает, что вы прочитали и согласны с пользовательским соглашением http://www . mathnet.ru/rus/agreement

Параметры загрузки:

IP: 35.173 .137 .237

26 апреля 2023 г., 14:46:35

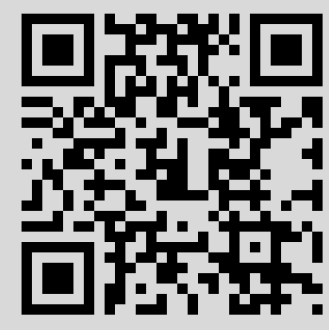




\section{Критерий невырожденности группы преобразований}

\section{В. А. Кыров}

1. Введение. Рассмотрим $n(n+1) / 2$-параметрическую локальную группу Ли локальных преобразований пространства $\mathbb{R}^{n}$ :

$$
x_{1}^{\prime}=\varphi_{1}\left(x_{1}, \ldots, x_{n}, a_{1}, \ldots, a_{n(n+1) / 2}\right), \quad \ldots, \quad x_{n}^{\prime}=\varphi_{n}\left(x_{1}, \ldots, x_{n}, a_{1}, \ldots, a_{n(n+1) / 2}\right),
$$

причем нулевым значениям параметров $a_{1}, \ldots, a_{n(n+1) / 2}$ соответствует тождественное преобразование. Базис ее алгебры Ли образуют [1] операторы

$$
X_{\mu}=\lambda_{\mu}^{1}\left(x_{1}, \ldots, x_{n}\right) \partial_{x_{1}}+\cdots+\lambda_{\mu}^{n}\left(x_{1}, \ldots, x_{n}\right) \partial_{x_{n}},
$$

где $\mu=1, \ldots, n(n+1) / 2$. Двухточечным инвариантом группы преобразований является интеграл системы дифференциальных уравнений [1]:

$$
X_{\mu}(i) f(i j)+X_{\mu}(j) f(i j)=0, \quad \mu=1, \ldots, \frac{n(n+1)}{2} .
$$

ОпреДЕЛЕниЕ. $n(n+1) / 2$-параметрическая локальная группа Ли локальных преобразований пространства $\mathbb{R}^{n}$ называется невырожденной, если ее двухточечный инвариант имеет открытую и плотную область определения $N \subset R^{2 n}$ и удовлетворяет условию невырожденности

$$
\frac{\partial\left(f\left(i i_{1}\right), \ldots, f\left(i i_{n}\right)\right)}{\partial\left(x_{1}(i), \ldots, x_{n}(i)\right)} \neq 0, \quad \frac{\partial\left(f\left(i_{1} i\right), \ldots, f\left(i_{n} i\right)\right)}{\partial\left(x_{1}(i), \ldots, x_{n}(i)\right)} \neq 0,
$$

где $x_{1}(i), \ldots, x_{n}(i)$ - координаты точки $i$, для открытого и плотного множества кортежей $n+1$ точек $\left\langle i i_{1} \ldots i_{n}\right\rangle$ и $\left\langle i_{1} \ldots i_{n} i\right\rangle$ из $\left(\mathbb{R}^{n}\right)^{n+1}$, причем пары $\left\langle i i_{1}\right\rangle, \ldots,\left\langle i i_{n}\right\rangle,\left\langle i_{1} i\right\rangle, \ldots,\left\langle i_{n} i\right\rangle \in$ $\mathbb{N}[2],[3]$.

В данной работе доказывается критерий, позволяющий по базисным операторам алгебры Ли установить невырожденность ей соответствующей локальной группы Ли локальных преобразований.

Теорема. Локальная $n(n+1) / 2$-параметрическая группа Ли локальных преобразований пространства $\mathbb{R}^{n}$ с базисными операторами (1) является невырожденной тогда и только тогда, когда

$$
\left|\begin{array}{cccc}
M_{11}\left(i i_{1}\right) & M_{12}\left(i i_{1}\right) & \cdots & M_{1 n}\left(i i_{1}\right) \\
M_{11}\left(i i_{2}\right) & M_{12}\left(i i_{2}\right) & \cdots & M_{1 n}\left(i i_{2}\right) \\
\cdots & \cdots & \cdots & \cdots \\
M_{11}\left(i i_{n}\right) & M_{12}\left(i i_{n}\right) & \cdots & M_{1 n}\left(i i_{n}\right)
\end{array}\right| \neq 0
$$

для открытого и плотного множества кортежей $n+1$ почек $\left\langle i i_{1} \ldots i_{n}\right\rangle$ из $\left(\mathbb{R}^{n}\right)^{n+1}$, пары $\left\langle i i_{1}\right\rangle, \ldots,\left\langle i i_{n}\right\rangle \in N, M_{11}, \ldots, M_{1 n}$ - миноры определителя

$$
\left|\begin{array}{cccccc}
z^{1} & \cdots & z^{n} & z^{n+1} & \cdots & z^{2 n} \\
\lambda_{1}^{1}(i) & \cdots & \lambda_{1}^{n}(i) & \lambda_{1}^{1}(j) & \cdots & \lambda_{1}^{n}(j) \\
\cdots & \cdots & \cdots & \cdots & \cdots & \cdots \\
\lambda_{2 n-1}^{1}(i) & \cdots & \lambda_{2 n-1}^{n}(i) & \lambda_{2 n-1}^{1}(j) & \cdots & \lambda_{2 n-1}^{n}(j)
\end{array}\right|,
$$

получаемые в пересечении 1-й строки и столбца под номером $k=1, \ldots, n$.

(C) В. А. Кыров, 2009 
Поставленная задача возникает в теории физических структур при классификации феноменологически симметричных геометрий, т.е. геометрий с невырожденными двухточечными инвариантами (метрическими функциями). Теорема позволяет из набора алгебр Ли $n(n+1) / 2$-параметрических групп Ли преобразований пространства $\mathbb{R}^{n}$ сразу выделить те, которые допускают невырожденный двухточечный инвариант (метрическая функция феноменологически симметричной, т.е. искомой геометрии).

2. Доказательство теоремы. Возьмем $n(n+1) / 2$-параметрическую локальную группу Ли локальных преобразований пространства $\mathbb{R}^{n}$ с базисными операторами (1). Уравнения инвариантности (2) записываются так:

$$
\lambda_{\mu}^{1}(i) \frac{\partial f(i j)}{\partial x_{1}(i)}+\cdots+\lambda_{\mu}^{n}(i) \frac{\partial f(i j)}{\partial x_{n}(i)}+\lambda_{\mu}^{1}(j) \frac{\partial f(i j)}{\partial x_{1}(j)}+\cdots+\lambda_{\mu}^{n}(j) \frac{\partial f(i j)}{\partial x_{n}(j)}=0,
$$

где $\mu=1, \ldots, n(n+1) / 2$. Матрица этой системы имеет размерность $(n(n+1) / 2) \times 2 n$, причем ее ранг равен $2 n-1$. Поэтому первые $2 n-1$ уравнений можно считать функционально независимыми, в противном случае необходимое число уравнений выделяется перенумерацией [2], [3]. Выделенная система $2 n-1$ уравнений в пространстве $\mathbb{R}^{2 n}$ координат $x_{1}(i) \ldots x_{n}(i) x_{1}(j) \ldots x_{n}(j)$ рассматривается как $2 n-1$ скалярных произведений

$$
\begin{gathered}
\left(\vec{M} \cdot \vec{F}_{1}\right)=0, \quad \ldots, \quad\left(\vec{M} \cdot \vec{F}_{2 n-1}\right)=0, \\
\vec{M}=\left(\frac{\partial f(i j)}{\partial x_{1}(i)}, \ldots, \frac{\partial f(i j)}{\partial x_{n}(i)}, \frac{\partial f(i j)}{\partial x_{1}(j)}, \ldots, \frac{\partial f(i j)}{\partial x_{n}(j)}\right), \\
\vec{F}_{\nu}=\left(\lambda_{\nu}^{1}(i), \ldots, \lambda_{\nu}^{n}(i), \lambda_{\nu}^{1}(j), \ldots, \lambda_{\nu}^{n}(j)\right),
\end{gathered}
$$

где $\nu=1, \ldots, 2 n-1$. Заметим, что векторы $\vec{F}_{1}, \ldots, \vec{F}_{2 n-1}$ линейно независимы в каждой точке некоторого открытого и плотного подмножества из $\mathbb{R}^{2 n}$, поскольку иначе им соответствующие базисные операторы из (1) будут линейно зависимыми в этом множестве, что недопустимо.

Рассмотрим $2 n-1$ векторов $\vec{a}_{1}=\left(a_{1}^{1}, \ldots, a_{1}^{2 n}\right), \ldots, \vec{a}_{2 n-1}=\left(a_{2 n-1}^{1}, \ldots, a_{2 n-1}^{2 n}\right)$ из $\mathbb{R}^{2 n}$. Под векторным произведением $2 n-1$ векторов $\vec{a}_{1}, \ldots, \vec{a}_{2 n-1}$ в $\mathbb{R}^{2 n}$ понимается вектор $\vec{a}=\left[\vec{a}_{1}, \ldots, \vec{a}_{2 n-1}\right]$ с координатами $\vec{a}=\left(a^{1}, \ldots, a^{2 n}\right): a^{\tau}=(-1)^{\tau+1} M_{1 \tau}$, где $\tau=1, \ldots, 2 n$, $M_{1 \tau}$ - минор определителя

$$
\left|\begin{array}{cccc}
a^{1} & a^{2} & \ldots & a^{2 n} \\
a_{1}^{1} & a_{1}^{2} & \ldots & a_{1}^{2 n} \\
\cdots & \ldots & \ldots & \ldots \\
a_{2 n-1}^{1} & a_{2 n-1}^{2} & \cdots & a_{2 n-1}^{2 n}
\end{array}\right| .
$$

Лемма. Векторное произведение $\vec{a}=\left[\vec{a}_{1}, \ldots, \vec{a}_{2 n-1}\right]$ линейно независимых векторов $\vec{a}_{1}=\left(a_{1}^{1}, \ldots, a_{1}^{2 n}\right), \ldots, \vec{a}_{2 n-1}=\left(a_{2 n-1}^{1}, \ldots, a_{2 n-1}^{2 n}\right)$ в пространстве $\mathbb{R}^{2 n}$ нормально $к$ гиперпространству, натянутому на эти векторы.

Вернемся снова к доказательству теоремы. Из леммы следует, что компоненты вектора $\vec{M}$ пропорциональны компонентам векторного произведения $\left[\vec{F}_{1}, \ldots, \vec{F}_{2 n-1}\right]$, значит,

$$
\begin{array}{llll}
\frac{\partial f(i j)}{\partial x_{1}(i)}=(-1)^{1+1} \omega M_{11}(i j), & \ldots, & \frac{\partial f(i j)}{\partial x_{n}(i)}=(-1)^{n+1} \omega M_{1 n}(i j), \\
\frac{\partial f(i j)}{\partial x_{1}(j)}=(-1)^{1+1} \omega M_{11}(j i), & \ldots, & \frac{\partial f(i j)}{\partial x_{n}(j)}=(-1)^{n+1} \omega M_{1 n}(j i),
\end{array}
$$

где $\omega=\omega\left(x_{1}(i), \ldots, x_{n}(i), x_{1}(j), \ldots, x_{n}(j)\right)$ - ненулевая гладкая функция, причем $M_{1 m}-$ минор определителя $\operatorname{det}\left|\vec{a}, \vec{F}_{1}, \ldots, \vec{F}_{2 n-1}\right|, m=1, \ldots, n$. Подставляя выше найденные производные в (3), приходим к (4). Таким образом, если группа Ли преобразований невырождена, т.е. выполняются неравенства (3), то имеет место неравенство (4) и наоборот. 
ПримеР. Рассмотрим группу движений евклидовой плоскости

$$
x^{\prime}=x \cos \alpha-y \sin \alpha+c, \quad y^{\prime}=x \cos \alpha+y \sin \alpha+d .
$$

Базис алгебры Ли образуют операторы $X_{1}=\partial_{x}, X_{2}=\partial_{y}, X_{3}=-y \partial_{x}+x \partial_{y}$. Очевидно, $M_{11}(i j)=x_{i}-x_{j}, M_{12}(i j)=y_{j}-y_{i}$. Тогда определитель неравенства (4) равен $\left(x_{k}-x_{i}\right) \times$ $\left(y_{j}-y_{i}\right)-\left(y_{k}-y_{i}\right)\left(x_{j}-x_{i}\right) \neq 0$. Таким образом, группа движений плоскости Евклида невырождена.

\section{СПИСОК ЦИТИРОВАННОЙ ЛИТЕРАТУРЫ}

[1] Л.В. Овсянников, Групповой анализ дифференциалъных уравнений, Наука, М., 1978. [2] Г. Г. Михайличенко, Докл. АН СССР, 269:2 (1983), 284-288. [3] Г. Г. Михайличенко, Полиметрические геометрии, Редакционно-издательский центр НГУ, Новосибирск, 2001.

\section{В. А. Кыров}

Поступило

Горно-Алтайский государственный университет (ГАГУ)

22.05.2008

E-mail: kfizika@gasu.ru 